\author{
for \\ the \\ communication \\ entitled
}

\title{
Total Syntheses of (+)-Cylindricines C-E and (-)-Lepadiformine Through a Common Intermediate Derived from an Aza-Prins Cyclization and Wharton's Rearrangement.
}

\author{
authored by
}

Jia Liu, Richard P. Hsung, * and Scott D. Peters

Department of Chemistry, University of Minnesota, Minneapolis, MN 55455 


\section{Amino Ketone 13.}

To a solution of $t$-BuLi $(16.0 \mathrm{~mL}, 1.5 \mathrm{M})$ in $\mathrm{Et}_{2} \mathrm{O}(2 \mathrm{~mL})$ was cannulated dropwise iodide $12(320.3 \mathrm{mg}, 11.0$ $\mathrm{mmol})$ in $\mathrm{Et}_{2} \mathrm{O}(1 \mathrm{~mL})$ at $-78{ }^{\circ} \mathrm{C}$. The solution was stirred at $-78{ }^{\circ} \mathrm{C}$ for $1 \mathrm{~h}$ and was added the solution of butyrolactam $11(4.45 \mathrm{~g}, 9.18 \mathrm{mmol})$ in THF $(5 \mathrm{~mL})$ at $-78{ }^{\circ} \mathrm{C}$. The mixture was stirred at $-78{ }^{\circ} \mathrm{C}$ for another $2 \mathrm{~h}$ and quenched with sat aq $\mathrm{NaHCO}_{3}$ at $-78{ }^{\circ} \mathrm{C}$. The mixture was slowly warmed up to rt and the aqueous fraction was extracted with $\mathrm{Et}_{2} \mathrm{O}$ [equal volume] for 3 times; The organic phases were combined, washed with sat aq $\mathrm{NaCl}$, dried over $\mathrm{Na}_{2} \mathrm{SO}_{4}$, and concentrated under reduced pressure. The residue was purified by flash column chromatography on silica gel (8\% EtOAc in hexanes) to provide 13 as a mixture of $E$ and $Z$ dienes in $61 \%$ yield $(3.89 \mathrm{~g})$.

$R_{f}=0.54(20 \%$ EtOAc in hexanes);

${ }^{1} \mathrm{H}$ NMR $\left(500 \mathrm{MHz}, \mathrm{CDCl}_{3}\right) \delta 7.65(\mathrm{~m}, 4 \mathrm{H}), 7.42(\mathrm{~m}, 6 \mathrm{H}), 6.06-5.96(\mathrm{~m}, 2 \mathrm{H}), 5.72-5.44(\mathrm{~m}, 2 \mathrm{H}), 4.68(\mathrm{br}, 1 \mathrm{H})$, 3.66-3.57 (m, 3H), $2.44(\mathrm{t}, J=7.5 \mathrm{~Hz}, 2 \mathrm{H}), 2.39(\mathrm{t}, J=7.0,2 \mathrm{H}), 2.19-2.16(\mathrm{~m}, 1 \mathrm{H}), 2.12-2.09(\mathrm{~m}, 1 \mathrm{H}), 2.06(\mathrm{~m}$, $2 \mathrm{H}), 1.81(\mathrm{~m}, 1 \mathrm{H}), 1.58(\mathrm{~m}, 1 \mathrm{H}), 1.45(\mathrm{~s}, 9 \mathrm{H}), 1.40-1.37(\mathrm{~m}, 4 \mathrm{H}), 1.32(\mathrm{~m}, 4 \mathrm{H}), 1.08(\mathrm{~s}, 9 \mathrm{H}), 0.89(\mathrm{t}, J=7.0 \mathrm{~Hz}$ $3 \mathrm{H}) ;{ }^{13} \mathrm{C} \mathrm{NMR}\left(125 \mathrm{MHz}, \mathrm{CDCl}_{3}\right) \delta 210.6,155.7,135.6,133.2,132.8,131.5,130.8,130.2,129.8,127.8,79.1$, 66.1, 51.7, 42.8, 39.3, 32.9, 32.4, 31.8, 29.4, 29.0, 28.9, 28.4, 26.9, 25.9, 23.4, 22.6, 19.3, 14.1; IR (film) $\mathrm{cm}^{-1}$ 3368brs, 3080w, 2930s, 1714s, 1498m, 1365m, 1172m, 1113s, 702m; mass spectrum (APCI): m/e (\%relative intensity) $548.4(\mathrm{M}+\mathrm{H}-\mathrm{Boc})^{+}(70), 530.3$ (100); m/e calcd for $\mathrm{C}_{40} \mathrm{H}_{61} \mathrm{NO}_{4} \mathrm{SiNa} 670.4262$, found 670.4270.

\section{Aza-Spirocycle 14.}

To a solution of 13 (2.34 g, $3.62 \mathrm{mmol})$ in the mixture of THF (1 mL) and toluene $(17 \mathrm{~mL})$ was added formic acid $(18 \mathrm{~mL})$ at $-10^{\circ} \mathrm{C}$. The solution was stirred at $-10^{\circ} \mathrm{C}$ [acetone/ice] for $3 \mathrm{~h}$. The mixture was quenched with sat aq $\mathrm{NaHCO}_{3}$ at $-10{ }^{\circ} \mathrm{C}$ [acetone/ice] and warmed up to rt. The aqueous fraction was extracted with $\mathrm{Et}_{2} \mathrm{O}$ [equal volume] for 3 times, and the organic phases were combined, washed with sat aq $\mathrm{NaCl}$, dried over $\mathrm{Na}_{2} \mathrm{SO}_{4}$, and concentrated under reduced pressure. The residue was purified by flash column chromatography on silica gel (6\% EtOAc in hexanes) to provide 14 as an inseparable diastereomeric mixture in $71 \%$ yield (1.46 g) based on the recovered starting material $13(0.37 \mathrm{~g})$.

$R_{f}=0.53(20 \%$ EtOAc in hexanes);

${ }^{1} \mathrm{H}$ NMR $\left(500 \mathrm{MHz}, \mathrm{CDCl}_{3}\right) \delta 8.05(\mathrm{~s}, 1 \mathrm{H}), 7.69-7.66(\mathrm{~m}, 4 \mathrm{H}), 7.41-7.37(\mathrm{~m}, 6 \mathrm{H}), 5.58(\mathrm{dd}, J=9.0,15.0 \mathrm{~Hz}$, $1 \mathrm{H}), 5.40(\mathrm{dd}, J=8.0,15.0 \mathrm{~Hz}, 1 \mathrm{H}), 5.32(\mathrm{~m}, 1 \mathrm{H}), 3.98(\mathrm{~m}, 1 \mathrm{H}), 3.67(\mathrm{dd}, J=3.5,9.5 \mathrm{~Hz}, 1 \mathrm{H}), 3.47(\mathrm{dd}, J=$ 9.5, 8.0 Hz, 1H), $3.37(\mathrm{~m}, 1 \mathrm{H}), 2.53(\mathrm{t}, J=12.5 \mathrm{~Hz}, 1 \mathrm{H}), 2.11(\mathrm{~m}, 1 \mathrm{H}), 1.94(\mathrm{~m}, 1 \mathrm{H}), 1.70-1.58(\mathrm{~m}, 7 \mathrm{H}), 1.37(\mathrm{~s}$ 9H), $1.32(\mathrm{~m}, 10 \mathrm{H}), 1.07(\mathrm{~s}, 9 \mathrm{H}), 0.89(\mathrm{t}, J=7.0 \mathrm{~Hz}, 3 \mathrm{H}) ;{ }^{13} \mathrm{C} \mathrm{NMR}\left(125 \mathrm{MHz}, \mathrm{CDCl}_{3}\right) \delta 160.5,152.9,136.6$, 135.6, 133.5, 129.7, 128.5, 127.7, 78.7, 75.3, 68.2, 64.7, 60.9, 42.3, 37.7, 34.6, 31.8, 31.3, 29.9, 29.1, 28.5, 26.9, 25.9, 25.1, 24.0, 22.7, 19.3, 14.1; IR (film) $\mathrm{cm}^{-1} 3076 \mathrm{w}, 2930 \mathrm{~s}, 2857 \mathrm{~s}, 1725 \mathrm{~s}, 1691 \mathrm{~s}, 1373 \mathrm{~s}, 1175 \mathrm{~s}, 1113 \mathrm{~s}$, $702 \mathrm{~m}$; mass spectrum (APCI): m/e (\%relative intensity) $576.3(\mathrm{M}+\mathrm{H}-\mathrm{Boc})^{+}(50), 530.3(100)$; m/e calcd for $\mathrm{C}_{41} \mathrm{H}_{61} \mathrm{NO}_{5} \mathrm{SiNa}$ 698.4211, found 698.4206. 


\section{Allylic Alcohol 15.}

To a solution of $14(1.45 \mathrm{~g}, 2.15 \mathrm{mmol})$ in the mixture of $\mathrm{MeOH}(10 \mathrm{~mL})$ and $\mathrm{H}_{2} \mathrm{O}(1 \mathrm{~mL})$ was added $\mathrm{K}_{2} \mathrm{CO}_{3}$ (138.2 $\mathrm{mg}, 6.75 \mathrm{mmol}$ ) at $0{ }^{\circ} \mathrm{C}$. The solution was stirred at $\mathrm{rt}$ for overnight followed by adding $20 \mathrm{~mL}^{\circ} \mathrm{H}_{2} \mathrm{O}$. The aqueous fraction was extracted with $\mathrm{CH}_{2} \mathrm{Cl}_{2}$ [equal volume] for 3 times, and the organic phases were combined, dried over $\mathrm{Na}_{2} \mathrm{SO}_{4}$, and concentrated under reduced pressure. The residue was purified by flash column chromatography on silica gel (with $6 \%$ and then with 10\% EtOAc in hexanes) to provide two diastereomeric isomers of $\mathbf{1 5}$ in $90 \%$ yield (1.25 g).

Isomer 1: $R_{f}=0.30(33 \%$ EtOAc in hexane $) ;[\alpha]^{23}=-61.3\left(\mathrm{c}=3.6, \mathrm{CHCl}_{3}\right)$;

${ }^{1} \mathrm{H}$ NMR $\left(500 \mathrm{MHz}, \mathrm{CDCl}_{3}\right) \delta 7.66(\mathrm{~m}, 4 \mathrm{H}), 7.44-7.37(\mathrm{~m}, 6 \mathrm{H}), 5.43(\mathrm{~m}, 2 \mathrm{H}), 4.01(\mathrm{~m}, 1 \mathrm{H}), 3.96(\mathrm{~m}, 1 \mathrm{H}), 3.66$ $(\mathrm{dd}, J=3.5,9.0,1 \mathrm{H}), 3.43(\mathrm{dd}, J=9.0,9.0 \mathrm{~Hz}, 1 \mathrm{H}), 3.34(\mathrm{~m}, 1 \mathrm{H}), 2.51(\mathrm{dt}, J=3.0,13.0 \mathrm{~Hz}, 1 \mathrm{H}), 2.10(\mathrm{dd}, J=$ 12.5, $8.0 \mathrm{~Hz}, 1 \mathrm{H}), 1.94(\mathrm{dd}, J=8.0,12.5 \mathrm{~Hz}, 1 \mathrm{H}), 1.77-1.75(\mathrm{~m}, 2 \mathrm{H}), 1.73-1.67(\mathrm{~m}, 4 \mathrm{H}), 1.48-1.41(\mathrm{~m}, 2 \mathrm{H})$, $1.36(\mathrm{~s}, 9 \mathrm{H}), 1.31(\mathrm{~m}, 10 \mathrm{H}), 1.23-1.18(\mathrm{~m}, 1 \mathrm{H}), 1.05(\mathrm{~s}, 9 \mathrm{H}), 0.88(\mathrm{t}, J=6.5 \mathrm{~Hz}, 3 \mathrm{H}) ;{ }^{13} \mathrm{C} \mathrm{NMR}(125 \mathrm{MHz}$, $\left.\mathrm{CDCl}_{3}\right) \delta 152.9,135.6,135.5,133.8,133.6,129.7,127.7,78.7,73.7,68.3,64.6,61.2,42.3,37.8,37.4,31.9$, $31.2,30.3,29.3,28.5,26.9,26.0,25.5,25.1,24.0,22.7,19.3,14.1$; IR (film) cm ${ }^{-1} 3440 \mathrm{br}, 3076 \mathrm{~m}, 2926 \mathrm{~s}, 2857 \mathrm{~s}$, $1692 \mathrm{~s}, 1389 \mathrm{~s}, 1172 \mathrm{~m}, 1112 \mathrm{~s}, 702 \mathrm{~m}$; mass spectrum (APCI): m/e (\%relative intensity) $548.3(\mathrm{M}+\mathrm{H}-\mathrm{Boc})^{+}(80)$, 530.3 (100); $\mathrm{m} / \mathrm{e}$ calcd for $\mathrm{C}_{40} \mathrm{H}_{61} \mathrm{NO}_{4} \mathrm{SiNa} 670.4262$, found 670.4267.

Isomer 2: $R_{f}=0.48(33 \%$ EtOAc in hexane $) ;[\alpha]^{23}=-66.8\left(\mathrm{c}=6.4, \mathrm{CHCl}_{3}\right)$;

${ }^{1} \mathrm{H}$ NMR $\left(500 \mathrm{MHz}, \mathrm{CDCl}_{3}\right) \delta 7.67(\mathrm{~m}, 4 \mathrm{H}), 7.44-7.37(\mathrm{~m}, 6 \mathrm{H}), 5.58-5.40(\mathrm{~m}, 2 \mathrm{H}), 4.07(\mathrm{~m}, 1 \mathrm{H}), 3.95(\mathrm{~m}, 1 \mathrm{H})$, $3.68(\mathrm{dd}, J=2.5,9.0,1 \mathrm{H}), 3.45(\mathrm{dd}, J=9.0,9.0 \mathrm{~Hz}, 1 \mathrm{H}), 3.34(\mathrm{~m}, 1 \mathrm{H}), 2.53(\mathrm{t}, J=12.0 \mathrm{~Hz}, 1 \mathrm{H}), 2.08(\mathrm{dd}, J=$ 12.5, $8.5 \mathrm{~Hz}, 1 \mathrm{H}), 1.94(\mathrm{dd}, J=8.5,12.5 \mathrm{~Hz}, 1 \mathrm{H}), 1.80-1.76(\mathrm{~m}, 2 \mathrm{H}), 1.70-1.66(\mathrm{~m}, 2 \mathrm{H}), 1.64-1.61(\mathrm{~m}, 2 \mathrm{H})$, $1.48(\mathrm{~m}, 2 \mathrm{H}), 1.36(\mathrm{~s}, 9 \mathrm{H}), 1.30(\mathrm{~m}, 10 \mathrm{H}), 1.23-1.18(\mathrm{~m}, 1 \mathrm{H}), 1.06(\mathrm{~s}, 9 \mathrm{H}), 0.89(\mathrm{t}, J=6.5 \mathrm{~Hz}, 3 \mathrm{H}) ;{ }^{13} \mathrm{C} \mathrm{NMR}$ $\left(125 \mathrm{MHz} \mathrm{CDCl}_{3}\right) \delta 153.0,135.6,135.6,133.6,132.3,129.6,127.7,78.7,72.7,68.5,64.7,61.1,42.0,37.8$, $37.4,31.9,31.3,30.0,29.4,28.6,26.9,26.0,25.3,25.1,24.0,22.7,19.3,14.1$; IR (film) cm $\mathrm{cm}^{-1} 3447 \mathrm{~b}, 3072 \mathrm{~m}$, 2924s, 2855s, 1677s, 1389s, 1406s, 1174m, 702m; mass spectrum (APCI): m/e (\%relative intensity) 548.3 $(\mathrm{M}+\mathrm{H}-\mathrm{Boc})^{+}(60), 530.3(100) ; \mathrm{m} / \mathrm{e}$ calcd for $\mathrm{C}_{40} \mathrm{H}_{61} \mathrm{NO}_{4} \mathrm{SiNa} 670.4262$, found 670.4267 .

\section{Enone 16.}

To a solution of $15(920.9 \mathrm{mg}, 1.42 \mathrm{mmol})$ in $\mathrm{CH}_{2} \mathrm{Cl}_{2}(7 \mathrm{~mL})$ was added 4-methylmorpholine $(249.5 \mathrm{mg}, 2.13$ mmol), molecular sieve $4 \AA$ (710.0 mg) and TPAP (tetrapropylammonium perruthenate) $(25.0 \mathrm{mg}, 0.071 \mathrm{mmol})$ at $\mathrm{rt}$. The solution was stirred at $\mathrm{rt}$ for $2 \mathrm{~h}$. The mixture was concentrated under reduced pressure and the residue was purified by flash column chromatography on silica gel (5\% EtOAc in hexanes) to provide 16 as colorless oil in $94 \%$ yield (860.9 $\mathrm{mg})$.

$R_{f}=0.50(20 \%$ EtOAc in hexane $) ;[\alpha]^{23}=-85.0\left(\mathrm{c}=5.40, \mathrm{CHCl}_{3}\right)$;

${ }^{1} \mathrm{H}$ NMR $\left(500 \mathrm{MHz}, \mathrm{CDCl}_{3}\right) \delta$ 7.66-7.64 (m, 4H), 7.43-7.38 (m, 6H), $6.70(\mathrm{dd}, J=8.0,16.0 \mathrm{~Hz}, 1 \mathrm{H}), 6.09(\mathrm{~d}, J$ 
$=16.0 \mathrm{~Hz}, 1 \mathrm{H}), 3.92(\mathrm{~m}, 1 \mathrm{H}), 3.65(\mathrm{dd}, J=3.5,10.0 \mathrm{~Hz}, 1 \mathrm{H}), 3.57(\mathrm{~m}, 1 \mathrm{H}), 3.45(\mathrm{dd}, J=7.5,10.0 \mathrm{~Hz}, 1 \mathrm{H})$, $2.55(\mathrm{dt}, J=3.5,13.0 \mathrm{~Hz}, 1 \mathrm{H}), 2.49$ (t, $J=7.5 \mathrm{~Hz}, 2 \mathrm{H}), 2.09(\mathrm{~m}, 1 \mathrm{H}), 1.94(\mathrm{~m}, 1 \mathrm{H}), 1.74-1.59(\mathrm{~m}, 8 \mathrm{H}), 1.36(\mathrm{~s}$, 9H), $1.30(\mathrm{~m}, 10 \mathrm{H}), 1.24(\mathrm{~m}, 1 \mathrm{H}), 1.05(\mathrm{~s}, 9 \mathrm{H}), 0.89(\mathrm{t}, J=6.5 \mathrm{~Hz}, 3 \mathrm{H}) ;{ }^{13} \mathrm{C} \mathrm{NMR}\left(125 \mathrm{MHz}, \mathrm{CDCl}_{3}\right) \delta 200.6$, 153.1, 148.1, 135.6, 133.4, 129.6, 127.7, 79.1, 68.4, 64.6, 61.0, 42.5, 41.1, 37.6, 31.7, 31.4, 29.1, 28.7, 28.5, 26.9, 25.8, 24.7, 24.4, 23.8, 22.5, 19.3, 14.1; IR (film) $\mathrm{cm}^{-1} 3076 \mathrm{w}, 2930 \mathrm{~s}, 2857 \mathrm{~s}, 1690 \mathrm{~s}, 1365 \mathrm{~s}, 1171 \mathrm{~m}, 1112 \mathrm{~s}$, $702 \mathrm{~m}$; mass spectrum (APCI): m/e (\%relative intensity) $546.3(\mathrm{M}+\mathrm{H}-\mathrm{Boc})^{+}(100), 528.3(20)$; m/e calcd for $\mathrm{C}_{40} \mathrm{H}_{59} \mathrm{NO}_{4} \mathrm{SiNa} 668.4111$, found 668.4098 .

\section{Epoxy Alcohol 19.}

To a solution of $15(1.44 \mathrm{~g}, 2.22 \mathrm{mmol})$ in $\mathrm{CH}_{2} \mathrm{Cl}_{2}(20 \mathrm{~mL})$ was added $m$-CPBA $(821 \mathrm{mg}, 3.33 \mathrm{mmol})$ at $-10{ }^{\circ} \mathrm{C}$. The solution was stirred at $\mathrm{rt}$ for overnight and was quenched with the mixture of sat aq $\mathrm{Na}_{2} \mathrm{~S}_{2} \mathrm{O}_{3}$ and sat aq $\mathrm{NaHCO}_{3}$. The aqueous fraction was extracted with $\mathrm{CH}_{2} \mathrm{Cl}_{2}$ [equal volume] for 3 times, and the organic phases were combined, dried over $\mathrm{Na}_{2} \mathrm{SO}_{4}$, concentrated under reduced pressure. The residue was purified by flash column chromatography on silica gel (8\% EtOAc in hexanes) to provide the diastereomeric mixture 19 as colorless oil in $94 \%$ yield $(1.514 \mathrm{~g})$.

$R_{f}=0.48(20 \%$ EtOAc in hexanes $)$;

${ }^{1} \mathrm{H}$ NMR $\left(500 \mathrm{MHz}, \mathrm{CDCl}_{3}\right) \delta$ 7.68-7.65 (m, 4H), 7.44-7.38 (m, 6H), $4.06(\mathrm{~m}, 1 \mathrm{H}), 3.74(\mathrm{~m}, 1 \mathrm{H}), 3.69(\mathrm{dd}, J=$ 3.5, $9.5 \mathrm{~Hz}, 1 \mathrm{H}), 3.48(\mathrm{dd}, J=9.5,9.5 \mathrm{~Hz}, 1 \mathrm{H}), 2.78(\mathrm{~m}, 1 \mathrm{H}), 2.46(\mathrm{~m}, 2 \mathrm{H}), 2.23(\mathrm{dd}, J=7.5,13.0 \mathrm{~Hz}, 1 \mathrm{H})$, $2.05(\mathrm{dd}, J=8.3,13.0 \mathrm{~Hz}, 1 \mathrm{H}), 1.93-1.85(\mathrm{~m}, 4 \mathrm{H}), 1.74(\mathrm{~m}, 1 \mathrm{H}), 1.63-1.58(\mathrm{~m}, 5 \mathrm{H}), 1.35(\mathrm{~s}, 9 \mathrm{H}), 1.44-1.22(\mathrm{~m}$, $10 \mathrm{H}), 1.07(\mathrm{~s}, 9 \mathrm{H}), 0.89(\mathrm{t}, J=6.5 \mathrm{~Hz}, 3 \mathrm{H}) ;{ }^{13} \mathrm{C} \mathrm{NMR}\left(125 \mathrm{MHz}, \mathrm{CDCl}_{3}\right) \delta 152.8,135.6,133.4,129.7,127.6$, 79.1, 68.9, 66.8, 64.4, 60.8, 60.3, 56.0, 41.7, 38.0, 33.6, 31.7, 31.4, 29.4, 28.5, 27.5, 26.9, 25.9, 25.6, 24.7, 23.8, 22.6, 19.3, 14.1; IR (film) $\mathrm{cm}^{-1} 3491 \mathrm{brs}, 3055 \mathrm{w}, 2928 \mathrm{~s}, 2857 \mathrm{~s}, 1690 \mathrm{~s}, 1472 \mathrm{~m}, 1373 \mathrm{~s}, 1172 \mathrm{~m}, 1106 \mathrm{~s}, 702 \mathrm{~m}$; mass spectrum (ESI): m/e (\%relative intensity) $686.4(\mathrm{M}+\mathrm{Na})^{+}(100) ; \mathrm{m} / \mathrm{e}$ calcd for $\mathrm{C}_{40} \mathrm{H}_{61} \mathrm{NO}_{5} \mathrm{SiNa}^{6} 686.4217$, found 686.4221 .

\section{Epoxy Ketone 20.}

To a solution of $19(1.51 \mathrm{~g}, 2.28 \mathrm{mmol})$ in $\mathrm{CH}_{2} \mathrm{Cl}_{2}(20 \mathrm{~mL})$ was added anhy DMSO $(1.78 \mathrm{~mL}, 25.1 \mathrm{mmol})$ and anhy $\mathrm{Et}_{3} \mathrm{~N}(1.60 \mathrm{~mL}, 11.5 \mathrm{mmol})$ at $\mathrm{rt}$. The solution was cooled to $-10{ }^{\circ} \mathrm{C}$ and was added $\mathrm{SO}_{3} \cdot \mathrm{pyridine}(1.45 \mathrm{~g}$, $9.11 \mathrm{mmol}$ ) in 3 portions. The solution was stirred at $\mathrm{rt}$ for overnight and was quenched with sat aq $\mathrm{NaCl}$ at -10 ${ }^{\circ} \mathrm{C}$. The aqueous fraction was extracted with $\mathrm{CH}_{2} \mathrm{Cl}_{2}$ [equal volume] for 3 times, and the combined organic phases were dried over $\mathrm{Na}_{2} \mathrm{SO}_{4}$ and concentrated in vacuo. The residue was purified by flash column chromatography on silica gel (4\% EtOAc in hexanes) to provide $\mathbf{2 0}$ as colorless oil in $80 \%$ yield (1.21 g).

$R_{f}=0.65(20 \%$ EtOAc in hexanes);

${ }^{1} \mathrm{H}$ NMR $\left(500 \mathrm{MHz}, \mathrm{CDCl}_{3}\right) \delta$ 7.68-7.67 (m, 4H), 7.62-7.37 (m, 6H), $4.05(\mathrm{~m}, 1 \mathrm{H}), 3.69(\mathrm{dd}, J=3.5,10.0 \mathrm{~Hz}$, $1 \mathrm{H}), 3.51(\mathrm{dd}, J=7.5,10.0 \mathrm{~Hz}, 1 \mathrm{H}), 3.29(\mathrm{~d}, J=2.0 \mathrm{~Hz}, 1 \mathrm{H}), 2.76(\mathrm{dd}, J=2.0,8.5 \mathrm{~Hz}, 1 \mathrm{H}), 2.56-2.42(\mathrm{~m}, 3 \mathrm{H})$, 
$2.32(\mathrm{~m}, 1 \mathrm{H}), 2.20(\mathrm{dd}, J=8.5,13.0 \mathrm{~Hz}, 1 \mathrm{H}), 2.02(\mathrm{dd}, J=8.0,13.0 \mathrm{~Hz}, 1 \mathrm{H}), 1.91(\mathrm{~m}, 1 \mathrm{H}), 1.79-1.72(\mathrm{~m}, 1 \mathrm{H})$, $1.60-1.58(\mathrm{~m}, 5 \mathrm{H}), 1.40(\mathrm{~s}, 9 \mathrm{H}), 1.31-1.28(\mathrm{~m}, 10 \mathrm{H}), 1.06(\mathrm{~s}, 9 \mathrm{H}), 0.87(\mathrm{t}, J=4.0 \mathrm{~Hz}, 3 \mathrm{H}) ;{ }^{13} \mathrm{C} \mathrm{NMR}(125 \mathrm{MHz}$ $\left.\mathrm{CDCl}_{3}\right) \delta 207.5,153.0,135.6,135.5,133.3,127.7,79.4,66.7,64.5,60.6,59.6,58.7,42.0,37.7,37.6,31.6,28.9$, $28.5,27.7,26.9,25.8,24.6,23.7,23.0,22.7,22.5,19.2,14.0$; IR (film) cm $\mathrm{cm}^{-1} 3063 \mathrm{w}, 2954 \mathrm{~s}, 2870 \mathrm{~s}, 1687 \mathrm{~s}$, $1468 \mathrm{~m}, 1366 \mathrm{~s}, 1170 \mathrm{~m}, 1112 \mathrm{~s}, 702 \mathrm{~m}$; mass spectrum (ESI): m/e (\%relative intensity) $684.4(\mathrm{M}+\mathrm{Na})^{+}(100) ; \mathrm{m} / \mathrm{e}$ calcd for $\mathrm{C}_{40} \mathrm{H}_{59} \mathrm{NO}_{5} \mathrm{SiNa} 684.4061$, found 684.4067 .

\section{Allylic Alcohol 23.}

To a solution of $20(1.37 \mathrm{~g}, 2.08 \mathrm{mmol})$ in $\mathrm{MeOH}(10 \mathrm{~mL})$ was added the solution of $95 \% \mathrm{NH}_{2} \mathrm{NH}_{2}(0.33 \mathrm{~mL}$, $10.4 \mathrm{mmol})$ in $5 \mathrm{~mL} \mathrm{MeOH}$ followed with $\mathrm{AcOH}(0.012 \mathrm{~mL}, 0.21 \mathrm{mmol})$ in $5 \mathrm{~mL} \mathrm{MeOH}$ dropwise at $-10^{\circ} \mathrm{C}$. The solution was stirred at $\mathrm{rt}$ for $2 \mathrm{~h}$ and was quenched with sat aq $\mathrm{NaHCO}_{3}$ at $-10{ }^{\circ} \mathrm{C}$. The aqueous fraction was extracted with $\mathrm{CH}_{2} \mathrm{Cl}_{2}$ [equal volume] for 3 times, and the organic phases were combined, dried over $\mathrm{Na}_{2} \mathrm{SO}_{4}$, and concentrated under reduced pressure. The residue was purified by flash column chromatography on silica gel (6\% EtOAc in hexanes) to provide an inseparable diastereomeric mixture 23 in $66 \%$ yield $(888.6 \mathrm{mg})$. $R_{f}=0.63(20 \%$ EtOAc in hexanes $)$;

${ }^{1} \mathrm{H}$ NMR $\left(500 \mathrm{MHz}, \mathrm{CDCl}_{3}\right) \delta$ 7.72-7.67 (m, 4H), 7.46-7.30 (m, 6H), 5.57-5.53 (m, 2H), 4.17-4.13 (m, 1H), $4.05(\mathrm{~m}, 1 \mathrm{H}), 3.73(\mathrm{dd}, J=3.6,9.5 \mathrm{~Hz}, 1 \mathrm{H}), 3.49(\mathrm{dd}, J=8.1,9.5 \mathrm{~Hz}, 1 \mathrm{H}), 2.72-2.36(\mathrm{~m}, 3 \mathrm{H}), 2.09-1.98(\mathrm{~m}$, $3 \mathrm{H}), 1.75-1.67(\mathrm{~m}, 7 \mathrm{H}), 1.35(\mathrm{~s}, 9 \mathrm{H}), 1.29(\mathrm{~m}, 10 \mathrm{H}), 1.08(\mathrm{~s}, 9 \mathrm{H}), 1.02(\mathrm{~m}, 1 \mathrm{H}), 0.90(\mathrm{t}, J=6.9 \mathrm{~Hz}, 3 \mathrm{H}) ;{ }^{13} \mathrm{C}$ NMR $\left(125 \mathrm{MHz}, \mathrm{CDCl}_{3}\right) \delta 152.9,135.6,133.6,131.2,130.4,130.0,127.7,78.7,71.3,68.2,64.8,61.4,44.4$, $39.1,32.3,31.8,29.4,28.9,28.7,28.5,26.9,25.7,24.9,24.6,23.0,22.7,19.3,14.2$; IR (film) cm $\mathrm{cm}^{-1} 3492 \mathrm{brs}$, 3079w, 2928s, 1684s, 1473m, 1365s, 1172m, 1112s; mass spectrum (ESI): m/e (\%relative intensity) 670.4 $(\mathrm{M}+\mathrm{Na})^{+}(100) ; \mathrm{m} / \mathrm{e}$ calcd for $\mathrm{C}_{40} \mathrm{H}_{61} \mathrm{NO}_{4} \mathrm{SiNa} 670.4268$, found 670.4267 .

\section{Enone 24.}

To a solution of $23(888.6 \mathrm{mg}, 1.37 \mathrm{mmol})$ in $\mathrm{CH}_{2} \mathrm{Cl}_{2}(15 \mathrm{~mL})$ was added $\mathrm{MnO}_{2}(2.38 \mathrm{~g}, 27.4 \mathrm{mmol})$ at rt. The solution was stirred at $\mathrm{rt}$ for $2 \mathrm{~d}$ and filtered through celite to remove the excess $\mathrm{MnO}_{2}$. The filtrate was concentrated under reduced pressure and the residue was purified by flash column chromatography on silica gel (4\% EtOAc in hexanes) to provide 24 as colorless oil in $90 \%$ yield (235 $\mathrm{mg}$ ) based on the recovered starting alcohol 23.

$R_{f}=0.69(20 \%$ EtOAc in hexanes $) ;[\alpha]^{23}=-127.6\left(\mathrm{c}=3.39, \mathrm{CHCl}_{3}\right)$;

${ }^{1} \mathrm{H}$ NMR $\left(500 \mathrm{MHz}, \mathrm{CDCl}_{3}\right) \delta 7.65(\mathrm{~m}, 4 \mathrm{H}), 7.42-7.37(\mathrm{~m}, 6 \mathrm{H}), 6.84(\mathrm{dt}, J=7.5,15.0 \mathrm{~Hz}, 1 \mathrm{H}), 6.16(\mathrm{~d}, J=$ $15.0 \mathrm{~Hz}, 1 \mathrm{H}), 3.98(\mathrm{dd}, J=12.3,3.3 \mathrm{~Hz}, 1 \mathrm{H}), 3.77(\mathrm{ddd}, J=3.3,7.5,7.5 \mathrm{~Hz}, 1 \mathrm{H}), 3.64(\mathrm{dd}, J=3.3,9.5 \mathrm{~Hz}$, $1 \mathrm{H}), 3.44(\mathrm{dd}, J=8.0,9.5 \mathrm{~Hz}, 1 \mathrm{H}), 2.66(\mathrm{ddd}, J=3.5,12.512 .5 \mathrm{~Hz}, 1 \mathrm{H}), 2.39(\mathrm{~m}, 1 \mathrm{H}), 2.16(\mathrm{~m}, 2 \mathrm{H}), 1.83(\mathrm{~m}$, $1 \mathrm{H}), 1.68-1.56(\mathrm{~m}, 5 \mathrm{H}), 1.47-1.42(\mathrm{~m}, 2 \mathrm{H}), 1.38(\mathrm{~s}, 9 \mathrm{H}), 1.35-1.23(\mathrm{~m}, 10 \mathrm{H}), 1.04(\mathrm{~s}, 9 \mathrm{H}), 0.87(\mathrm{t}, J=7.0 \mathrm{~Hz}$ $3 \mathrm{H}) ;{ }^{13} \mathrm{C}$ NMR $\left(125 \mathrm{MHz}, \mathrm{CDCl}_{3}\right) \delta 203.9,153.3,146.2,135.5,133.4,130.4,129.7,127.7,79.1,68.0,64.4$, 
60.6, 49.8, 38.6, 32.5, 31.7, 31.3, 29.0, 28.6, 28.5, 26.9, 26.6, 24.9, 24.4, 23.7, 22.6, 19.2, 14.1; IR (film) $\mathrm{cm}^{-1}$ 3080w, 2928s, 2855s, 1689s, 1621m, 1373s, 1106s, 702m; mass spectrum (ESI): m/e (\%relative intensity) 668.4 $(\mathrm{M}+\mathrm{Na})^{+}(100) ; \mathrm{m} / \mathrm{e}$ calcd for $\mathrm{C}_{40} \mathrm{H}_{59} \mathrm{NO}_{4} \mathrm{SiNa} 668.4111$, found 668.4115.

\section{Common Intermediate Aza-Tricycle 26.}

To a solution of $24(235.0 \mathrm{mg}, 0.364 \mathrm{mmol})$ in $\mathrm{CH}_{2} \mathrm{Cl}_{2}(1.8 \mathrm{~mL})$ was added TFA $(1.8 \mathrm{~mL})$ dropwise at $-10{ }^{\circ} \mathrm{C}$. The solution was stirred at $-10{ }^{\circ} \mathrm{C}$ for $2 \mathrm{~h}$ and was quenched with sat aq $\mathrm{NaHCO}_{3}$ at $-10{ }^{\circ} \mathrm{C}$. The aqueous fraction was quickly extracted with $\mathrm{CH}_{2} \mathrm{Cl}_{2}$ [equal volume] for 3 times, the organic phases were combined, dried over $\mathrm{Na}_{2} \mathrm{SO}_{4}$, and concentrated under reduced pressure to provide 26 in $72 \%$ yield (143.2 mg) as a sufficiently pure sample for the next step.

$R_{f}=0.56(25 \%$ EtOAc in hexanes $) ;[\alpha]_{\mathrm{D}}^{23}=-53.1\left(\mathrm{c}=0.35, \mathrm{CHCl}_{3}\right)$;

${ }^{1} \mathrm{H}$ NMR (500 MHz, $\left.\mathrm{CDCl}_{3}\right) \delta$ 7.68-7.64 (m, 4H), 7.43-7.36 (m, 6H), $3.74(\mathrm{dd}, J=4.5,9.5 \mathrm{~Hz}, 1 \mathrm{H}), 3.47(\mathrm{~m}$, $1 \mathrm{H}), 3.28(\mathrm{dd}, J=9.5,9.5 \mathrm{~Hz}, 1 \mathrm{H}), 3.18(\mathrm{~m}, 1 \mathrm{H}), 2.62(\mathrm{dd}, J=3.0,11.0 \mathrm{~Hz}, 1 \mathrm{H}), 2.16-2.14(\mathrm{~m}, 2 \mathrm{H}), 2.11-2.05$ (m, 1H), 2.01-1.94 (m, 1H), $1.91(\mathrm{~m}, 1 \mathrm{H}), 1.81$ (ddd, $J=3.5,6.5,12.5 \mathrm{~Hz}, 1 \mathrm{H}), 1.75-1.52(\mathrm{~m}, 5 \mathrm{H}), 1.42(\mathrm{ddd}, J$ $=3.5,12.5 \mathrm{~Hz}, 1 \mathrm{H}), 1.36-1.17(\mathrm{~m}, 6 \mathrm{H}), 1.17-1.07(\mathrm{~m}, 6 \mathrm{H}), 1.08(\mathrm{~s}, 9 \mathrm{H}), 0.85(\mathrm{t}, J=7.0 \mathrm{~Hz}, 3 \mathrm{H}) ;{ }^{13} \mathrm{C} \mathrm{NMR}(125$ $\left.\mathrm{MHz}, \mathrm{CDCl}_{3}\right) \delta 213.4,135.6,133.9,129.6,127.6,70.4,68.3,60.3,54.7,54.0,41.5,40.8,33.4,31.7,31.5,29.2$, 28.3, 27.3, 26.9, 25.0, 24.1, 22.6, 22.6, 19.3, 14.0; IR (film) $\mathrm{cm}^{-1} 3069 \mathrm{~m}, 2933 \mathrm{~s}, 2857 \mathrm{~s}, 1705 \mathrm{~s}, 1471 \mathrm{~m}, 1428 \mathrm{~m}$, $1261 \mathrm{~s}, 1106 \mathrm{~s}$; mass spectrum (ESI): m/e (\%relative intensity) $546.4(\mathrm{M}+\mathrm{H})^{+}$(100); m/e calcd for $\mathrm{C}_{35} \mathrm{H}_{52} \mathrm{NO}_{2} \mathrm{Si}$ 546.3769 , found 546.3766 .

\section{Epimerization Product 27.}

To a solution of $26(3.1 \mathrm{mg}, 0.0057 \mathrm{mmol})$ in $\mathrm{MeOH}(1 \mathrm{~mL})$ was added $\mathrm{K}_{2} \mathrm{CO}_{3}(7.8 \mathrm{mg}, 0.057 \mathrm{mmol})$ at rt. The solution was stirred at $\mathrm{rt}$ for overnight and to this reaction mixture was added $5 \mathrm{~mL}$ of $\mathrm{H}_{2} \mathrm{O}$. The aqueous fraction was extracted with $\mathrm{CH}_{2} \mathrm{Cl}_{2}$ [equal volume] for 3 times, and the organic phases were combined, dried over $\mathrm{Na}_{2} \mathrm{SO}_{4}$, and concentrated under reduced pressure to provide 27 in 97\% yield (3.0 mg) as a sufficiently pure sample.

$R_{f}=0.70(25 \%$ EtOAc in hexane $) ;[\alpha]^{23}=14.3\left(\mathrm{c}=0.42, \mathrm{CHCl}_{3}\right)$;

${ }^{1} \mathrm{H}$ NMR $\left(500 \mathrm{MHz}, \mathrm{CDCl}_{3}\right) \delta$ 7.74-7.68 (m, 4H), 7.68-7.36 (m, 6H), $3.63(\mathrm{dd}, J=2.4,8.4 \mathrm{~Hz}, 1 \mathrm{H}), 3.36(\mathrm{~m}$, $1 \mathrm{H}), 3.30(\mathrm{dd}, J=8.4,8.4 \mathrm{~Hz}, 1 \mathrm{H}), 3.10(\mathrm{~m}, 1 \mathrm{H}), 2.30-2.09(\mathrm{~m}, 7 \mathrm{H}), 1.68-1.64(\mathrm{~m}, 1 \mathrm{H}), 1.55-1.48(\mathrm{~m}, 3 \mathrm{H})$, 1.34-1.28 (m, 9H), $1.09(\mathrm{~s}, 9 \mathrm{H}), 1.09-1.04(\mathrm{~m}, 5 \mathrm{H}), 0.86(\mathrm{t}, J=7.2 \mathrm{~Hz}, 3 \mathrm{H}) ;{ }^{13} \mathrm{C} \mathrm{NMR}\left(125 \mathrm{MHz} \mathrm{CDCl}_{3}\right) \delta$ 211.4, 135.5, 133.9, 129.5, 127.5, 70.1, 68.2, 57.6, 55.2, 50.8, 42.8, 35.7, 34.8, 34.6, 31.5, 28.9, 26.8, 26.7, 25.7, 24.2, 22.8, 22.4, 21.8, 19.1, 13.9; IR (film) $\mathrm{cm}^{-1} 3076 \mathrm{~m}, 2937 \mathrm{~s}, 2861 \mathrm{~s}, 1708 \mathrm{~s}, 1466 \mathrm{~m}, 1262 \mathrm{~m}, 1112 \mathrm{~s}$; mass spectrum (ESI): m/e (\%relative intensity) $546.4(\mathrm{M}+\mathrm{H})^{+}(100) ; \mathrm{m} / \mathrm{e}$ calcd for $\mathrm{C}_{35} \mathrm{H}_{52} \mathrm{NO}_{2} \mathrm{Si} 546.3769$, found 546.3766. 


\section{(+)-Cylindricine C Ent-1c.}

To a solution of $26(45.0 \mathrm{mg}, 0.082 \mathrm{mmol})$ in THF $(1 \mathrm{~mL})$ was added TBAF $(0.16 \mathrm{~mL}, 0.16 \mathrm{mmol})$ at $-10{ }^{\circ} \mathrm{C}$. The solution was stirred at $\mathrm{rt}$ for overnight and to this reaction mixture was added $5 \mathrm{~mL}$ of $\mathrm{H}_{2} \mathrm{O}$. The aqueous fraction was extracted with $\mathrm{Et}_{2} \mathrm{O}$ [equal volume] for 3 times, and the organic phases were combined, washed with sat aq $\mathrm{NaCl}$, dried over $\mathrm{Na}_{2} \mathrm{SO}_{4}$, and concentrated under reduced pressure. The residue was purified by flash column chromatography on silica gel (70\% EtOAc in hexanes) to provide $(+)$-cylindricine $\mathrm{C}$ ent-1c as pale yellow oil in $91 \%$ yield $(22.9 \mathrm{mg})$.

$R_{f}=0.39$ (in EtOAc); $[\alpha]^{23}{ }_{\mathrm{D}}=59.8\left(\mathrm{c}=1.72, \mathrm{CHCl}_{3}\right)\left(\right.$ literature $[$ Trost $]:[\alpha]^{25}{ }_{\mathrm{D}}=60.8\left(\mathrm{c}=0.4, \mathrm{CH}_{2} \mathrm{Cl}_{2}\right)$ ${ }^{1} \mathrm{H}$ NMR (500 MHz, $\left.\mathrm{CDCl}_{3}\right) \delta 3.53(\mathrm{~m}, 2 \mathrm{H}), 3.43(\mathrm{~d}, J=9.5 \mathrm{~Hz}, 1 \mathrm{H}), 3.29(\mathrm{~m}, 1 \mathrm{H}), 2.90(\mathrm{br}, 1 \mathrm{H}), 2.31(\mathrm{t}, J=$ $12.5 \mathrm{~Hz}, 2 \mathrm{H}), 2.24(\mathrm{dd}, J=2.8,13.2 \mathrm{~Hz}, 2 \mathrm{H}), 2.12(\mathrm{dd}, J=7.8,12.3 \mathrm{~Hz}, 1 \mathrm{H}), 1.84$ (ddd, $J=2.0,8.5,13.5 \mathrm{~Hz}$, $1 \mathrm{H}), 1.71-1.59(\mathrm{~m}, 5 \mathrm{H}), 1.49(\mathrm{~m}, 1 \mathrm{H}), 1.44-1.27(\mathrm{~m}, 13 \mathrm{H}), 0.88(\mathrm{t}, J=7.0 \mathrm{~Hz}, 3 \mathrm{H}) ;{ }^{13} \mathrm{C} \mathrm{NMR}(125 \mathrm{MHz}$, $\left.\mathrm{CDCl}_{3}\right) \delta 210.5,70.7,66.4,56.6,55.3,50.3,42.6,36.5,35.9,35.2,31.7,29.3,28.8,27.1,24.3,22.8,22.6,21.9$, 14.1; IR (film) cm $\mathrm{cm}^{-1} 3436 \mathrm{br}, 2932 \mathrm{~s}, 2857 \mathrm{~m}, 1705 \mathrm{~s}, 1448 \mathrm{~m}, 1040 \mathrm{~m}$; mass spectrum (ESI): m/e (\%relative intensity) $308.3(\mathrm{M}+\mathrm{H})^{+}(100)$; m/e calcd for $\mathrm{C}_{19} \mathrm{H}_{34} \mathrm{NO}_{2} 308.2591$, found 308.2578.

\section{(+)-Cylindricine D Ent-1d.}

To a solution of ent-1c $(11.1 \mathrm{mg}, 0.036 \mathrm{mmol})$ in $\mathrm{CH}_{3} \mathrm{CN}(0.5 \mathrm{~mL})$ was added $\mathrm{Ag}_{2} \mathrm{O}(167.04 \mathrm{mg}, 0.72 \mathrm{mmol})$ and $\mathrm{MeI}(0.11 \mathrm{~mL}, 1.8 \mathrm{mmol})$ at $\mathrm{rt}$. The solution was stirred at $\mathrm{rt}$ for $3 \mathrm{~d}$ and filtered through celite to remove the excess $\mathrm{Ag}_{2} \mathrm{O}$. The filtrate was concentrated under reduced pressure and the residue was purified by flash column chromatography on silica gel $(6 \%$ EtOAc in hexanes) to provide $(+)$-cylindricine D ent-1d as pale yellow oil in $44 \%$ yield $(22.9 \mathrm{mg})$.

$R_{f}=0.39$ (in 20\% EtOAc in hexanes);

$[\alpha]_{\mathrm{D}}^{23}=21.8\left(\mathrm{c}=0.57, \mathrm{CHCl}_{3}\right)$ (literature [Trost $]:[\alpha]_{\mathrm{D}}^{25}=21.5\left(\mathrm{c}=0.08, \mathrm{CH}_{2} \mathrm{Cl}_{2}\right)$

${ }^{1} \mathrm{H}$ NMR $\left(500 \mathrm{MHz}, \mathrm{CDCl}_{3}\right) \delta 3.42(\mathrm{~m}, 1 \mathrm{H}), 3.38(\mathrm{~m}, 1 \mathrm{H}), 3.37(\mathrm{~s}, 3 \mathrm{H}), 3.21(\mathrm{~m}, 1 \mathrm{H}), 3.05(\mathrm{dd}, J=9.0,9.0 \mathrm{~Hz}$, $1 \mathrm{H}), 2.28-2.17(\mathrm{~m}, 3 \mathrm{H}), 2.12-2.02(\mathrm{~m}, 2 \mathrm{H}), 1.686(\mathrm{~m}, 1 \mathrm{H}), 1.74-1.56(\mathrm{~m}, 5 \mathrm{H}), 1.49-1.39(\mathrm{~m}, 2 \mathrm{H}), 1.36-1.25(\mathrm{~m}$, 12H), $0.89(\mathrm{t}, J=7.0 \mathrm{~Hz}, 3 \mathrm{H}) ;{ }^{13} \mathrm{C}$ NMR $\left(125 \mathrm{MHz}, \mathrm{CDCl}_{3}\right) \delta 211.3,78.2,70.1,59.1,55.5,55.5,50.9,42.9$,

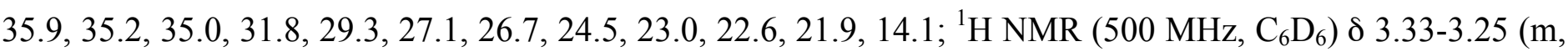
2H), $3.14(\mathrm{~s}, 3 \mathrm{H}), 3.10-3.08(\mathrm{~m}, 1 \mathrm{H}), 2.96(\mathrm{dd}, J=8.5,8.5 \mathrm{~Hz}, 1 \mathrm{H}), 2.36(\mathrm{dm}, J=13.0 \mathrm{~Hz}, 1 \mathrm{H}), 2.11(\mathrm{dm}, 1 \mathrm{H})$, 1.89-1.85 (m, 3H), $1.74(\mathrm{~m}, 2 \mathrm{H}), 1.63-1.52(\mathrm{~m}, 4 \mathrm{H}), 1.45-1.39(\mathrm{~m}, 2 \mathrm{H}), 1.33-1.12(\mathrm{~m}, 12 \mathrm{H}), 0.86(\mathrm{t}, J=7.0 \mathrm{~Hz}$,

$3 \mathrm{H}) ;{ }^{13} \mathrm{C}$ NMR $\left(75 \mathrm{MHz}, \mathrm{C}_{6} \mathrm{D}_{6}\right) \delta 209.1,79.0,70.1,59.1,56.1,55.8,51.2,43.4,36.6,35.8,35.5,32.5,30.0$, 27.7, 27.3, 25.2, 23.7, 23.3, 22.8, 14.7;

IR (film) cm $\mathrm{cm}^{-1} 2929 \mathrm{~s}, 2861 \mathrm{~s}, 1708 \mathrm{~s}, 1450 \mathrm{~m}, 1330 \mathrm{~m}, 1194 \mathrm{~m}, 1116 \mathrm{~s}$; mass spectrum (ESI): m/e (\%relative intensity) $308.3(\mathrm{M}+\mathrm{H})^{+}(100)$; m/e calcd for $\mathrm{C}_{20} \mathrm{H}_{36} \mathrm{NO}_{2} 322.2741$, found 322.2738. 
(+)-Cylindricine E Ent-1e. To a solution of ent-1c $(2.1 \mathrm{mg}, 0.0068 \mathrm{mmol})$ in $\mathrm{CH}_{2} \mathrm{Cl}_{2}(0.5 \mathrm{~mL})$ was added $\mathrm{Et}_{3} \mathrm{~N}$ $(0.021 \mathrm{~mL}, 0.15 \mathrm{mmol})$, DMAP $(0.12 \mathrm{mg}, 14.4 \mathrm{~mol} \%)$ and acetic anhydride $(9.3 \mu \mathrm{L}, 0.098 \mathrm{mmol})$ at $-10{ }^{\circ} \mathrm{C}$. The solution was stirred at $\mathrm{rt}$ for $2 \mathrm{~h}$ and quenched with $\mathrm{H}_{2} \mathrm{O}$ at $-10{ }^{\circ} \mathrm{C}$. The aqueous fraction was extracted with $\mathrm{CH}_{2} \mathrm{Cl}_{2}$ [equal volume] for 3 times, and the organic phases were combined, dried over $\mathrm{Na}_{2} \mathrm{SO}_{4}$, and concentrated under reduced pressure. The residue was purified by flash column chromatography on silica gel (5\% EtOAc in hexane) to provide (+)-cylindricine E ent-1e in $50 \%$ yield $(1.7 \mathrm{mg})$.

$R_{f}=0.45$ (33\% EtOAc in hexane);

$[\alpha]^{23}{ }_{D}=27.5\left(\mathrm{c}=0.16, \mathrm{CHCl}_{3}\right)$ (literature [Trost $]:[\alpha]_{\mathrm{D}}^{25}=28.67\left(\mathrm{c}=0.13, \mathrm{CH}_{2} \mathrm{Cl}_{2}\right)$

${ }^{1} \mathrm{H}$ NMR $\left(500 \mathrm{MHz}, \mathrm{CDCl}_{3}\right) \delta 4.12(\mathrm{dd}, J=2.8,10.5 \mathrm{~Hz}, 1 \mathrm{H}), 4.68(\mathrm{dd}, J=9.0,10.5 \mathrm{~Hz}, 1 \mathrm{H}), 3.52(\mathrm{ddd}, J=$ 3.0, 8.3, 8.3 Hz, 1H), 3.22 (dddd, $J=5.8,8.5,8.5,8.5 \mathrm{~Hz}, 1 \mathrm{H}), 2.25-2.21(\mathrm{~m}, 4 \mathrm{H}), 2.13-2.05(\mathrm{~m}, 1 \mathrm{H}), 2.08(\mathrm{~s}$, $3 \mathrm{H}), 1.81-1.75(\mathrm{~m}, 2 \mathrm{H}), 1.69-1.64(\mathrm{~m}, 2 \mathrm{H}), 1.64-1.56(\mathrm{~m}, 1 \mathrm{H}), 1.49-1.45(\mathrm{~m}, 2 \mathrm{H}), 1.36-1.27(\mathrm{~m}, 13 \mathrm{H}), 0.88(\mathrm{t}, J$ $=7.0 \mathrm{~Hz}, 3 \mathrm{H}) ;{ }^{13} \mathrm{C} \mathrm{NMR}\left(75 \mathrm{MHz}, \mathrm{CDCl}_{3}\right) \delta$ 210.9, 171.0, 70.0, 68.7, 55.3, 54.6, 51.1, 43.0, 36.1, 35.0, 31.9, 29.4, 27.2, 26.5, 24.5, 23.1, 22.7, 22.0, 21.2, 14.2; IR (film) $\mathrm{cm}^{-1} 2934 \mathrm{~s}, 2860 \mathrm{~s}, 1742 \mathrm{~s}, 1707 \mathrm{~s}, 1448 \mathrm{~m}, 1381 \mathrm{~m}$, $1227 \mathrm{~s}$; mass spectrum (ESI): m/e (\%relative intensity) $350.3(\mathrm{M}+\mathrm{H})^{+}(100) ; \mathrm{m} / \mathrm{e}$ calcd for $\mathrm{C}_{21} \mathrm{H}_{56} \mathrm{NO}_{3} 350.2697$, found 350.2695 .

\section{Xantate 28.}

To a solution of 26 (198.5 mg, $0.364 \mathrm{mmol})$ in $\mathrm{MeOH}(4 \mathrm{~mL})$ was added $\mathrm{NaBH}_{4}(137.7 \mathrm{mg}, 3.64 \mathrm{mmol})$ at -10 ${ }^{\circ} \mathrm{C}$. The solution was stirred at $\mathrm{rt}$ for $2 \mathrm{~h}$ and was quenched with $\mathrm{H}_{2} \mathrm{O}$. The aqueous fraction was extracted with $\mathrm{CH}_{2} \mathrm{Cl}_{2}$ [equal volume] for 3 times, and the organic phases were combined, dried over $\mathrm{Na}_{2} \mathrm{SO}_{4}$, and concentrated under reduced pressure. The residue was purified by flash column chromatography on silica gel $(100 \%$ EtOAc) to provide alcohol in $100 \%$ yield $(198.0 \mathrm{mg})$.

$R_{f}=0.34(\mathrm{EtOAc}+10 \% \mathrm{MeOH}) ;[\alpha]_{\mathrm{D}}^{23}=-7.12\left(\mathrm{c}=1.77, \mathrm{CHCl}_{3}\right)$

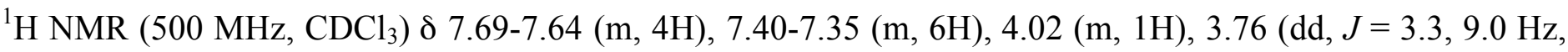
1H), $3.28(\mathrm{dd}, J=9.0,9.0 \mathrm{~Hz}, 1 \mathrm{H}), 3.26(\mathrm{~m}, 1 \mathrm{H}), 2.82(\mathrm{~m}, 1 \mathrm{H}), 2.20(\mathrm{~m}, 1 \mathrm{H}), 2.03(\mathrm{~m}, 2 \mathrm{H}), 1.77(\mathrm{~m}, 2 \mathrm{H}), 1.69$ $1.55(\mathrm{~m}, 5 \mathrm{H}), 1.49-1.36(\mathrm{~m}, 4 \mathrm{H}), 1.34-1.22(\mathrm{~m}, 5 \mathrm{H}), 1.16-1.07(\mathrm{~m}, 5 \mathrm{H}), 1.05(\mathrm{~s}, 9 \mathrm{H}), 0.97(\mathrm{~m}, 1 \mathrm{H}), 0.85(\mathrm{t}, J=$ $7.0 \mathrm{~Hz}, 3 \mathrm{H}) ;{ }^{13} \mathrm{C}$ NMR $\left(75 \mathrm{MHz}, \mathrm{CDCl}_{3}\right) \delta 135.6,134.0,129.5,127.6,70.8,69.8,60.2,53.6,43.5,41.0,33.1$, 31.9, 30.4, 29.5, 29.0, 27.6, 27.0, 26.5, 26.1, 24.4, 22.7, 19.4, 14.2; IR (film) cm $\mathrm{cm}^{-1} 3401 \mathrm{br}, 3072 \mathrm{w}, 2930 \mathrm{~s}, 2858 \mathrm{~s}$, $1651 \mathrm{~m}, 1463 \mathrm{~m}, 1428 \mathrm{~m}, 1113 \mathrm{~s}$; mass spectrum (ESI): m/e (\%relative intensity) $639.4(\mathrm{M}+\mathrm{H})^{+}(100) ; \mathrm{m} / \mathrm{e}$ calcd for $\mathrm{C}_{35} \mathrm{H}_{54} \mathrm{NO}_{2} \mathrm{Si} 548.3918$, found 548.3925.

To a solution of the alcohol $(198.0 \mathrm{mg}, 0.364 \mathrm{mmol})$ in THF $(5 \mathrm{~mL})$ was added $\mathrm{NaH}(29.1 \mathrm{mg}, 0.728 \mathrm{mmol})$ at $10{ }^{\circ} \mathrm{C}$. The solution was stirred at $\mathrm{rt}$ for $1 \mathrm{~h}$ and to this solution was added $\mathrm{CS}_{2}(0.109 \mathrm{~mL}, 1.82 \mathrm{mmol})$ and stirred for another $2 \mathrm{~h}$. The mixture was added MeI $(0.227 \mathrm{~mL}, 3.64 \mathrm{mmol})$ and stirred at rt for overnight. The reaction was quenched with $\mathrm{H}_{2} \mathrm{O}$ and the aqueous fraction was extracted with $\mathrm{Et}_{2} \mathrm{O}$ [equal volume] for 3 times. 
The organic phases were combined, washed with sat aq $\mathrm{NaCl}$, dried over $\mathrm{Na}_{2} \mathrm{SO}_{4}$, and concentrated under reduced pressure. The residue was purified by flash column chromatography on silica gel (10\% EtOAc in hexane) to provide the xantate $\mathbf{2 8}$ in $72 \%$ yield (167.0 $\mathrm{mg}$ ).

$R_{f}=0.67(20 \%$ EtOAc in Hexane $) ;[\alpha]^{23}=-21.9\left(\mathrm{c}=1.04, \mathrm{CHCl}_{3}\right)$

${ }^{1} \mathrm{H}$ NMR $\left(500 \mathrm{MHz}, \mathrm{CDCl}_{3}\right) \delta$ 7.68-7.64 (m, 4H), 7.40-7.27 (m, 6H), $5.80(\mathrm{ddd}, J=5.5,5.5,10.8 \mathrm{~Hz}, 1 \mathrm{H}), 3.71$ $(\mathrm{dd}, J=4.0,9.5 \mathrm{~Hz}, 1 \mathrm{H}), 3.29(\mathrm{dd}, J=9.5,9.5 \mathrm{~Hz}, 1 \mathrm{H}), 3.24(\mathrm{~m}, 1 \mathrm{H}), 2.86(\mathrm{~m}, 1 \mathrm{H}), 2.56(\mathrm{~s}, 3 \mathrm{H}), 2.32(\mathrm{~m}, 1 \mathrm{H})$, $2.13(\mathrm{~m}, 1 \mathrm{H}), 1.97(\mathrm{~m}, 1 \mathrm{H}), 1.91(\mathrm{~m}, 1 \mathrm{H}), 1.83-1.73(\mathrm{~m}, 3 \mathrm{H}), 1.50-1.07(\mathrm{~m}, 17 \mathrm{H}), 1.08(\mathrm{~s}, 9 \mathrm{H}), 0.96(\mathrm{~m}, 1 \mathrm{H})$

$0.85(\mathrm{t}, J=7.0 \mathrm{~Hz}, 3 \mathrm{H}) ;{ }^{13} \mathrm{C} \mathrm{NMR}\left(125 \mathrm{MHz}, \mathrm{CDCl}_{3}\right) \delta$ 215.6, 135.6, 134.1, 129.5, 127.6, 83.0, 70.6, 66.6, 60.0, 52.8, 43.1, 42.4, 33.6, 31.8, 31.2, 30.8, 29.5, 28.6, 27.6, 27.0, 26.4, 24.3, 22.7, 19.3, 19.0, 14.1; IR (film) $\mathrm{cm}^{-1} 3071 \mathrm{w}, 2930 \mathrm{~s}, 2857 \mathrm{~s}, 1471 \mathrm{~m}, 1428 \mathrm{~m}, 1216 \mathrm{~s}, 1056 \mathrm{~s}$; mass spectrum (ESI): m/e (\%relative intensity) 638.4 $(\mathrm{M}+\mathrm{H})^{+}(100) ; \mathrm{m} / \mathrm{e}$ calcd for $\mathrm{C}_{37} \mathrm{H}_{56} \mathrm{NO}_{2} \mathrm{~S}_{2} \mathrm{Si} 638.3516$, found 638.3516.

\section{(-)-Lepadiformine 4.}

To a solution of $n \mathrm{Bu}_{3} \mathrm{SnH}(0.044 \mathrm{~mL}, 0.103 \mathrm{mmol})$ in benzene $(1.5 \mathrm{~mL})$ was slowly added the solution of xantate 28 (21.8 $\mathrm{mg}, 0.0342 \mathrm{mmol})$ and AIBN (3.6 mg, $0.0137 \mathrm{mmol})$ in benzene $(0.5 \mathrm{~mL})$ through a syringe pump in $5 \mathrm{~h}$ at $80{ }^{\circ} \mathrm{C}$. The solvent was removed under reduced pressure and the residue was purified by flash column chromatography on silica gel (40\% EtOAc in hexane) to provide the deoxygenation product as pale yellow oil in $100 \%$ yield (18.2 $\mathrm{mg})$.

$R_{f}=0.29(33 \%$ EtOAc in hexanes $) ;[\alpha]^{23}=-20.0\left(\mathrm{c}=0.22, \mathrm{CHCl}_{3}\right)$

${ }^{1} \mathrm{H}$ NMR $\left(500 \mathrm{MHz}, \mathrm{CDCl}_{3}\right) \delta 7.65(\mathrm{~m}, 4 \mathrm{H}), 7.37(\mathrm{~m}, 6 \mathrm{H}), 3.75(\mathrm{dd}, J=4.0,9.5 \mathrm{~Hz}, 1 \mathrm{H}), 3.26(\mathrm{dd}, J=9.5,9.0$ $\mathrm{Hz}, 1 \mathrm{H}), 3.21(\mathrm{~m}, 1 \mathrm{H}), 2.97(\mathrm{~m}, 1 \mathrm{H}), 2.09-2.05$ (m, 2H), 1.68-1.53 (m, 6H), $1.44(\mathrm{~m}, 4 \mathrm{H}), 1.37-1.20(\mathrm{~m}, 10 \mathrm{H})$, 1.20-1.09 (m, 4H), $1.05(\mathrm{~s}, 9 \mathrm{H}), 0.91(\mathrm{~m}, 1 \mathrm{H}), 0.85(\mathrm{t}, J=7.0 \mathrm{~Hz}, 3 \mathrm{H}) ;{ }^{13} \mathrm{C} \mathrm{NMR}\left(125 \mathrm{MHz}, \mathrm{CDCl}_{3}\right) \delta 135.6$, 134.2, 129.5, 127.6, 71.2, 67.1, 59.7, 53.5, 41.4, 37.9, 34.3, 31.8, 30.9, 29.5, 29.1, 27.9, 27.6, 27.0, 26.4, 24.5, 24.0, 22.7, 19.3, 14.1; IR (film) $\mathrm{cm}^{-1} 3071 \mathrm{w}, 2929 \mathrm{~s}, 2857 \mathrm{~s}, 1693 \mathrm{w}, 1463 \mathrm{~m}, 1337 \mathrm{~m}, 1112 \mathrm{~s}$; mass spectrum (ESI): $\mathrm{m} / \mathrm{e}\left(\%\right.$ relative intensity) $532.4(\mathrm{M}+\mathrm{H})^{+}(100) ; \mathrm{m} / \mathrm{e}$ calcd for $\mathrm{C}_{35} \mathrm{H}_{54} \mathrm{NOSi} 532.3969$, found 532.3967.

To a solution of the deoxygenation product $(18.2 \mathrm{mg}, 0.0342 \mathrm{mmol})$ in THF $(0.5 \mathrm{~mL})$ was added TBAF $(0.035$ $\mathrm{mL}, 0.035 \mathrm{mmol}$ ) at $-10{ }^{\circ} \mathrm{C}$. The solution was stirred at $\mathrm{rt}$ for overnight and was quenched with $\mathrm{H}_{2} \mathrm{O}$. The aqueous fraction was extracted with $\mathrm{Et}_{2} \mathrm{O}$ [equal volume] for 3 times, and the organic phases were combined, washed with sat aq $\mathrm{NaCl}$, dried over $\mathrm{Na}_{2} \mathrm{SO}_{4}$, and concentrated under reduced pressure. The residue was purified by flash column chromatography on silica gel $\left(\mathrm{CHCl}_{3}+2 \% \mathrm{MeOH}+1 \% \mathrm{NH}_{4} \mathrm{OH}\right)$ to provide (-)lepadiformine 4 in $79 \%$ yield $(8.0 \mathrm{mg})$.

$R_{f}=0.34(20 \% \mathrm{MeOH}$ in EtOAc);

$[\alpha]_{\mathrm{D}}^{23}=-14.3(\mathrm{c}=0.30, \mathrm{MeOH})\left(\right.$ literature [Kibayash]: $[\alpha]_{\mathrm{D}}^{25}=-15.0(\mathrm{c}=0.37, \mathrm{MeOH})$

${ }^{1} \mathrm{H}$ NMR (500 MHz, $\left.\mathrm{CDCl}_{3}\right) \delta 3.40(\mathrm{~m}, 1 \mathrm{H}), 3.35(\mathrm{~m}, 1 \mathrm{H}), 3.25(\mathrm{dd}, J=9.5,9.5 \mathrm{~Hz}, 1 \mathrm{H}), 3.17(\mathrm{~m}, 1 \mathrm{H}), 1.81-$ 
$1.46(\mathrm{~m}, 13 \mathrm{H}), 1.37-1.14(\mathrm{~m}, 14 \mathrm{H}), 1.06-0.98(\mathrm{~m}, 1 \mathrm{H}), 0.88(\mathrm{t}, J=7.0 \mathrm{~Hz}, 3 \mathrm{H}) ;{ }^{13} \mathrm{C} \mathrm{NMR}\left(125 \mathrm{MHz}, \mathrm{CDCl}_{3}\right) \delta$ 68.0, 62.0, 58.7, 53.6, 39.6, 38.1, 33.8, 31.8, 30.5, 29.6, 27.9, 27.5, 27.5, 26.2, 24.2, 23.2, 22.6, 22.3, 14.1; IR (film) $\mathrm{cm}^{-1} 3403 \mathrm{br}, 2919 \mathrm{~s}, 2853 \mathrm{~s}, 1463 \mathrm{~m}, 1081 \mathrm{~m}$; mass spectrum (ESI): $\mathrm{m} / \mathrm{e}$ (\%relative intensity) 294.3 $(\mathrm{M}+\mathrm{H})^{+}(100) ; \mathrm{m} / \mathrm{e}$ calcd for $\mathrm{C}_{19} \mathrm{H}_{36} \mathrm{NO} 294.2797$, found 294.2790 .

\section{${ }^{1}$ H NMR SPECTROSCOPIC COMPARISONS}

\section{H NMR Spectroscopic}

Comparisons:

\section{Cylindricine C}

Isolation

$3.49(1 \mathrm{H})$

$3.45(1 \mathrm{H})$

$3.35(1 \mathrm{H})$

$3.22(1 \mathrm{H})$

$2.25(4 \mathrm{H})$

$2.2(1 \mathrm{H})$

$2.10(2 \mathrm{H})$

$1.75(1 \mathrm{H})$

$1.68(1 \mathrm{H})$

$1.65(1 \mathrm{H})$

$1.5(1 \mathrm{H})$

$1.4(1 \mathrm{H})$

$1.38(2 \mathrm{H})$

$1.36(1 \mathrm{H})$

$1.35(1 \mathrm{H})$

$1.30(9 \mathrm{H})$

$0.90(3 \mathrm{H})$
Molander

$3.51(2 \mathrm{H})$

$3.41(1 \mathrm{H})$

$3.26(1 \mathrm{H})$

$2.85(1 \mathrm{H})$

$2.28(2 \mathrm{H})$

$2.21(2 \mathrm{H})$

$2.10(1 \mathrm{H})$

$1.81(1 \mathrm{H})$

1.70-1.17 (19H)

$0.85(3 \mathrm{H})$
Observed $\quad \Delta \mathrm{d}$ (Isolation) $\quad \Delta \mathrm{d}$ (Molander)

$3.53(2 \mathrm{H})$

0.04

0.02

$3.43(1 \mathrm{H})$

$3.29(1 \mathrm{H})$

0.08

0.02

0.07

0.03

$2.90(1 \mathrm{H})$

$2.31(2 \mathrm{H})$

0.05

$2.24(2 \mathrm{H})$

$2.12(1 \mathrm{H})$

$1.84(1 \mathrm{H})$

0.06

0.03

0.03

$-0.08$

0.02

$1.71-1.59(5 \mathrm{H})$

$1.49(1 \mathrm{H})$

$1.44-1.27(13 \mathrm{H})$

$0.88(3 \mathrm{H})$

$-0.02$

0.03 
Isolation

paper)

$3.61(1 \mathrm{H})$

$3.55(3 \mathrm{H})$

$3.42(1 \mathrm{H})$

$3.38(1 \mathrm{H})$

$3.26(1 \mathrm{H})$

$2.40(1 \mathrm{H})$

$2.16(2 \mathrm{H})$

$2.12(1 \mathrm{H})$

$2.00(1 \mathrm{H})$

$1.88(1 \mathrm{H})$

$1.82(3 \mathrm{H})$

$1.76(1 \mathrm{H})$

$1.70(2 \mathrm{H})$

$1.50(3 \mathrm{H})$

$1.46(5 \mathrm{H})$

$1.42(2 \mathrm{H})$

$1.18(3 \mathrm{H})$

$1.10(2 \mathrm{H})$

\section{Cylindricine D}

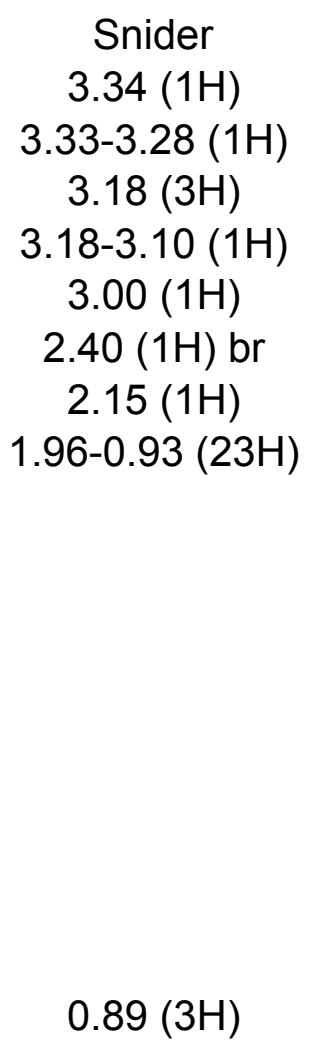

\begin{tabular}{|c|c|c|}
\hline $\begin{array}{c}\text { Observed } \\
3.33-3.25(2 \mathrm{H})\end{array}$ & $\Delta \mathrm{d}$ (Isolation) & $\begin{array}{c}\Delta \mathrm{d} \text { (Snider) } \\
\quad-0.05\end{array}$ \\
\hline $3.14(3 \mathrm{H})$ & & -0.04 \\
\hline $3.10-3.08(1 \mathrm{H})$ & & -0.05 \\
\hline $2.96(1 \mathrm{H})$ & & -0.04 \\
\hline $2.36(1 \mathrm{H})$ & & -0.04 \\
\hline $2.19-2.10(1 \mathrm{H})$ & & \\
\hline $\begin{array}{c}1.89-1.85(3 \mathrm{H}) \\
1.74(2 \mathrm{H})\end{array}$ & & \\
\hline $1.63-1.52(4 \mathrm{H})$ & & \\
\hline $\begin{array}{c}1.45-1.39(2 \mathrm{H}) \\
1.33-1.12 \\
(12 \mathrm{H})\end{array}$ & & \\
\hline
\end{tabular}

$0.86(3 \mathrm{H})$

$-0.03$ 


\section{Isolation}

Paper

$5.39(1 \mathrm{H}) \mathrm{br}$

$\begin{array}{cc}4.13(1 \mathrm{H}) & 4.21(1 \mathrm{H}) \\ 3.25(1 \mathrm{H}) & 3.68(1 \mathrm{H}) \\ ? & 3.55-3.45(1 \mathrm{H}) \\ & 3.28-3.16(1 \mathrm{H}) \\ 2.31-2.0(6 \mathrm{H}) & 2.28-2.14(3 \mathrm{H}) \\ & 2.07(3 \mathrm{H}) \\ & 1.84-1.04(22)\end{array}$

1.80-1.46

$(20 \mathrm{H})$

$0.88(3 \mathrm{H})$

2.04-1.01 (28
H)

$1.02(1 \mathrm{H})$

$0.87(3 \mathrm{H})$

Kibayashi

3.37-3.15

$(3 \mathrm{H})$

Weinreb

3.37-3.30 (2H)

$3.19(1 \mathrm{H})$

3.13-3.10 (1H)

$1.78-1.13(27 \mathrm{H})$

1.04-0.97 $(1 \mathrm{H})$

$0.85(3 \mathrm{H})$
Snider

Observed

$4.12(1 \mathrm{H})$

$3.68(1 \mathrm{H})$

$3.52(1 \mathrm{H})$

$3.22(1 \mathrm{H})$

2.25-2.01 (4H)

2.13-2.05 (1H)

$2.08(3 \mathrm{H})$

$$
\begin{gathered}
1.81-1.75(2 \mathrm{H}) \\
1.69-1.64(2 \mathrm{H}) \\
1.64-1.56(1 \mathrm{H}) \\
1.49-1.45(2 \mathrm{H}) \\
1.36-1.27
\end{gathered}
$$

$(13 \mathrm{H})$

$0.88(3 \mathrm{H})$
$\Delta \mathrm{d}$ (Isolation) $\Delta \mathrm{d}$ (Snider)

$\begin{array}{cc}0.01 & 0.03 \\ 0.43 & 0.03 \\ ? & 0.03 \\ & 0.03 \\ 0.06 & 0 \\ & 0 \\ & 0.03\end{array}$

0.01

0.01

0.01

0.04

0.03

\section{Lepadiformine}

Observed $\quad \Delta \mathrm{d}$ (Kibayashi) (Weinreb)

$\begin{array}{lll}3.40(1 \mathrm{H}) & 0.03 & 0.03\end{array}$

$3.35(1 \mathrm{H})$

$3.25(1 \mathrm{H})$

0.06

$3.17(1 \mathrm{H})$

1.81-1.46

0.04

$(13 \mathrm{H})$

1.37-1.14

$(14 \mathrm{H})$

$\begin{array}{lll}1.06-0.98(1 \mathrm{H}) & 0.04 & 0.02\end{array}$

$\begin{array}{lll}0.88(3 \mathrm{H}) & 0.01 & 0.03\end{array}$ 


\section{${ }^{13}$ C NMR SPECTROSCOPIC COMPARISONS}

13C NMR Spectroscopic

Comparisons:

Kibayashi

62

58.9

53.7

37.9

33.5

31.8

30.6

29.7

29.5

27.3

26.1

24.1

23.2

22.6

14.1

?

\section{Lepadiformine}

Weinreb

67.4

62.4

58.4

53.3

40.2

38.3

34.1

31.8

30.5

29.6

28.2

27.7

27.6

26.3

24.3

23.3

22.7

22.6

14
Observed

68

62

58.7

53.6

39.6

38.1

33.8

31.8

30.5

29.6

27.9

27.5

27.5

26.2

24.2

23.2

22.6

22.3

14.1
$\Delta \mathrm{d}$

?

$\Delta \mathrm{d}$ (Weinreb)

0.6

$-0.4$

0.3

0.3

$-0.6$

$-0.2$

$-0.3$

0

0

0

$-0.3$

$-0.2$

$-0.1$

$-0.1$

$-0.1$

$-0.1$

$-0.1$

$-0.3$

$-0.1$ 


\section{Isolation}

Paper

211

71.2

66.9

57.2

55.9

50.8

43.1

36.9

36.5

35.7

32.2

29.8

29.2

27.6

24.8

23.3

23.1

22.4

14.5
Trost

210.6

70.7

66.4

56.6

55.4

50.3

42.6

36.5

36

35.2

31.7

29.4

28.8

27.2

24.3

22.8

22.6

21.9

14.1
Cylindricine C

$\begin{array}{ccc}\text { Observed } & \Delta \mathrm{d} \text { (Isolation) } & \Delta \mathrm{d} \text { (Trost) } \\ 210.5 & -0.5 & -0.1 \\ 70.7 & -0.5 & 0 \\ 66.4 & -0.5 & 0 \\ 56.6 & -0.6 & 0 \\ 55.3 & -0.6 & -0.1 \\ 50.3 & -0.5 & 0 \\ 42.6 & -0.5 & 0 \\ 36.5 & -0.4 & 0 \\ 35.9 & -0.6 & -0.1 \\ 35.2 & -0.5 & 0 \\ 31.7 & -0.5 & 0 \\ 29.3 & -0.5 & -0.1 \\ 28.8 & -0.4 & 0 \\ 27.1 & -0.5 & -0.1 \\ 24.3 & -0.5 & 0 \\ 22.8 & -0.5 & 0 \\ 22.6 & -0.5 & 0 \\ 21.9 & -0.5 & 0 \\ 14.1 & -0.4 & 0\end{array}$




$\begin{array}{cc}\text { Isolation } & \\ \text { Paper } & \text { Snider } \\ 209.5 & 209.1 \\ 79.3 & 79 \\ 70.3 & 70.1 \\ 59.4 & 59.1 \\ 56.4 & 56.2 \\ 56.1 & 55.8 \\ 51.6 & 51.3 \\ 43.7 & 43.5 \\ 36.9 & 36.7 \\ 36 & 35.8 \\ 35.8 & 35.5 \\ 32.8 & 32.5 \\ 30.3 & 30.1 \\ 28 & 27.7 \\ 27.6 & 27.3 \\ 25.5 & 25.2 \\ 24 & 23.7 \\ 23.6 & 23.4 \\ 23.1 & 22.8 \\ 14.9 & 14.7\end{array}$

Cylindricine D

$\begin{array}{ccc}\text { Observed } & 0 \mathrm{~d} \text { (Isolation) } & \Delta \mathrm{d} \text { (Snider) } \\ 209.1 & -0.4 & 0 \\ 79 & -0.3 & 0 \\ 70.1 & -0.2 & 0 \\ 59.1 & -0.3 & 0 \\ 56.1 & -0.3 & -0.1 \\ 55.8 & -0.3 & 0 \\ 51.2 & -0.4 & -0.1 \\ 43.4 & -0.3 & -0.1 \\ 36.6 & -0.3 & -0.1 \\ 35.8 & -0.2 & 0 \\ 35.5 & -0.3 & 0 \\ 32.5 & -0.3 & 0 \\ 30 & -0.3 & -0.1 \\ 27.7 & -0.3 & 0 \\ 27.3 & -0.3 & 0 \\ 25.2 & -0.3 & 0 \\ 23.7 & -0.3 & 0 \\ 23.3 & -0.3 & -0.1 \\ 22.8 & -0.3 & 0 \\ 14.7 & -0.2 & 0\end{array}$




$\begin{array}{cc}\text { Isolation } & \\ \text { Paper } & \text { Snider } \\ 211.8 & 211 \\ 171.5 & 171 \\ 70.8 & 70 \\ 69.4 & 68.6 \\ 56 & 55.2 \\ 55.3 & 54.5 \\ 51.9 & 51.1 \\ 43.7 & 42.9 \\ 36.8 & 36 \\ 35.7 & 34.9 \\ 32.6 & 31.8 \\ 27.7 & 29.3 \\ 27.2 & 27.1 \\ 25.2 & 26.4 \\ 23.7 & 24.4 \\ 23.3 & 22.9 \\ 22.7 & 22.6 \\ 21.8 & 21.9 \\ 21.8 & 21 \\ 14.8 & 14\end{array}$

Cylindricine E

$\begin{array}{ccc}\text { Observed } & \Delta \mathrm{d} \text { (Isolation) } & \Delta \mathrm{d} \text { (Snider) } \\ 210.9 & -0.9 & -0.1 \\ 171 & -0.5 & 0 \\ 70 & -0.8 & 0 \\ 68.7 & -0.7 & 0.1 \\ 55.3 & -0.7 & 0.1 \\ 54.6 & -0.7 & 0.1 \\ 51.1 & -0.8 & 0 \\ 43 & -0.7 & 0.1 \\ 36.1 & -0.7 & 0.1 \\ 35 & -0.7 & 0.1 \\ 31.9 & -0.7 & 0.1 \\ 29.4 & 1.7 & 0.1 \\ 27.2 & 0 & 0.1 \\ 26.5 & 1.1 & 0.1 \\ 24.5 & 0.8 & 0.1 \\ 23.1 & -0.2 & 0.2 \\ 22.7 & 0 & 0.1 \\ 22 & 0.2 & 0.1 \\ 21.2 & -0.6 & 0.2 \\ 14.2 & -0.6 & 0.2\end{array}$




\section{${ }^{1}$ H AND ${ }^{13}$ C NMR SPECTROSCOPIC COMPARISONS FOR COMPOUND 26}

$\begin{array}{ccc}\text { Trost } & \text { Compound 26 } & \Delta \mathrm{d} \\ 7.64(4 \mathrm{H}) & 7.68-7.64(4 \mathrm{H}) & \\ 7.36(6 \mathrm{H}) & 7.43-7.36(6 \mathrm{H}) & \\ 3.71(1 \mathrm{H}) & 3.74(1 \mathrm{H}) & 0.03 \\ 3.44(1 \mathrm{H}) & 3.46(1 \mathrm{H}) & 0.02 \\ 3.25(1 \mathrm{H}) & 3.28(1 \mathrm{H}) & 0.03 \\ 3.14(1 \mathrm{H}) & 3.18(1 \mathrm{H}) & 0.02 \\ 2.60(1 \mathrm{H}) & 2.62(1 \mathrm{H}) & 0.03 \\ 2.13(2 \mathrm{H}) & 2.16-2.14(2 \mathrm{H}) & \\ 1.98-1.03 & 2.11-2.05(1 \mathrm{H}) & \\ (22 \mathrm{H}) & 2.01-1.94(1 \mathrm{H}) & \\ & 1.91(1 \mathrm{H}) & \\ & 1.81(1 \mathrm{H}) & \\ & 1.75-1.52(5 \mathrm{H}) & \\ & 1.42(1 \mathrm{H}) & 0.06 \\ & 1.36-1.17(6 \mathrm{H}) & \end{array}$




$\begin{array}{ccc}\text { Trost } & \text { Compound 26 } & \Delta \mathrm{d} \\ 213.6 & 213.4 & -0.2 \\ 135.7 & 135.6 & \\ 134 & 133.9 & \\ 133.9 & 129.6 & \\ 129.7 & 127.6 & \\ 129.6 & & \\ 127.7 & & 0 \\ 70.4 & 70.4 & 0 \\ 68.3 & 68.3 & 0 \\ 60.3 & 60.3 & 0 \\ 54.7 & 54.7 & -0.1 \\ 54.1 & 54 & 0 \\ 41.5 & 41.5 & 0.1 \\ 40.7 & 40.8 & 0 \\ 33.4 & 33.4 & -0.1 \\ 31.8 & 31.7 & 0 \\ 31.5 & 31.5 & -0.1 \\ 29.3 & 29.2 & -0.1 \\ 28.4 & 28.3 & 0 \\ 27.3 & 27.3 & 0 \\ 26.9 & 26.9 & 0 \\ 25 & 25 & -0.1 \\ 24.2 & 24.1 & -0.1 \\ 22.7 & 22.6 & 0 \\ 22.6 & 22.6 & 0 \\ 19.3 & 19.3 & \\ 14.1 & 14 & \end{array}$

